\title{
Investigation of the Spatial Clustering Properties of Seismic Time Series: A Comparative Study from Shallow to Intermediate-Depth Earthquakes
}

\author{
Ke Ma ${ }^{1},{ }^{1}$ Long Guo, ${ }^{1}$ and Wangheng Liu $^{2}$ \\ ${ }^{1}$ School of Mathematics and Physics, China University of Geosciences, Wuhan 430074, China \\ ${ }^{2}$ Department of Mathematics, Michigan State University, East Lansing, MI 48824, USA \\ Correspondence should be addressed to Ke Ma; make@cug.edu.cn
}

Received 17 July 2018; Accepted 16 October 2018; Published 1 November 2018

Guest Editor: David Cuesta-Frau

Copyright (C) $2018 \mathrm{Ke} \mathrm{Ma}$ et al. This is an open access article distributed under the Creative Commons Attribution License, which permits unrestricted use, distribution, and reproduction in any medium, provided the original work is properly cited.

\begin{abstract}
In this paper, a size-independent modification of the general detrended fluctuation analysis (DFA) method is introduced. With this modified DFA, seismic time series $(m \geq 4.5)$ pertaining to most seismically active regions of the world from the year 1972 up to the year 2016 are comparatively analyzed. An eminent homogeneity of spatial clustering behaviors in worldwide range is detected and DFA scaling exponents coincide with previous results for local regions. Furthermore, universal nontrivial spatial clustering behaviors are revealed from shallow to intermediate-depth earthquakes by varying the depth of the hypocenter: in shallow earthquakes, the depth range corresponding to the minimum spatial clustering is first verified to be spatiotemporal and identical; in intermediate-depth earthquakes, strong weakening long-range correlations compared to shallow earthquakes are unveiled to strengthen the recent findings in seismology. Our work aims to suggest a possible statistical approach to explore the dynamic mechanisms implied in the seismicity as well as in other analogous dynamic evolution processes.
\end{abstract}

\section{Introduction}

Among multifarious natural hazards, seismicity is a representative complex spatiotemporal phenomenon, in which underlying mechanisms are not yet fully understood $[1$, 2]. Statistics analyzing provides important insights into fundamental properties of seismic processes, such as the well-known Gutenberg-Richter laws [3] for the magnitude distribution of earthquakes and the Omori laws [4] for the time evolution of the frequency of aftershocks. Inspired by these fractal analogies, Bak has invoked the self-organized criticality (SOC) concept [5] into seismicity. For reviews on this topic, see [6-8] and references therein.

Usually, in statistical analysis, earthquakes can be treated as spatiotemporal stochastic point processes $[9,10]$ due to their nonhomogeneous occurrence time. In such processes, spatial and temporal clustering behaviors featured by correlations come to light and develop into important properties of seismic activity [11-20]. In seismology, such correlations originate from multifarious microscopic dynamics, governing complex interactions of many components over a worldwide range of time or space scales. It is known that an earthquake can trigger the subsequent one even more than $1000 \mathrm{~km}$ far away $[21,22]$, implying that the long-range correlations exist. In such a situation, identifying the properties of long-range correlations may shed light on the fundamental dynamic seismic mechanisms. This approach has been continuously attracting seismologists, geologists, and physicists' attentions [22-31].

However, identifying long-range correlations in seismicity is usually still a tricky business mainly due to the irregular fluctuations and severe nonstationarity embedded in the emitted seismic signals $[25,32,33]$. Such nonstationarity is of a much larger order than that of any other known natural signal. Peng et al. [34] propose the DFA as a robust method which can provide a quantitative representation of the correlation properties of a series of signals. The advantage of DFA over many other methods is that it permits the detection of long-range correlations embedded in seemingly nonstationary time series and also avoids the spurious 
detection of apparent long-range correlation which is an artifact of nonstationarity. In seismology, the earthquakes can be described as points represented by the geophysical coordinates (longitude and latitude) in space and a sequence of points distributed for time axis where such temporal points are determined by their occurrence time. Accordingly, the seismic time series can be initialized by the spatial or temporal interval between subsequent earthquakes for the analysis of seismic mechanisms $[13,27,29,35-38]$. With the aid of DFA analysis on these series, scaling behaviors of fluctuations can be explored to qualify the earthquakes correlation characteristics. Already, there have been many works performed in this approach: Telesca has analyzed the spatiotemporal clustering behavior in earthquakes, especially the dependence of the time-clustering behavior on the depth $(0-30 \mathrm{~km})$ of the earthquakes in southern California (SC) [36]; Z. Y Zheng et al. have found DFA scaling exponent of interval time series has no dependence on hypocenter depth or epicentral distance in Japan earthquakes [25]; P. A Varotsos and N. V Sarlis have discovered that the minima earthquake preceding violent earthquake is followed by characteristic changes of temporal correlations between earthquake magnitudes [39]. Other related results can be found in [40-44].

Recently, the studies of the global earthquake network strengthen the idea that the Earth behaves as a self-organized critical system with long spatial correlations between earthquakes $[45,46]$. This means the earthquake spatial clustering may be not only regional but also global. Nevertheless, to the best of our knowledge, most of the current works which are probing earthquakes spatial clustering with DFA method have been carried out in the absence of worldwide comparative study. In this work, a comparative DFA analysis will be performed to explore the possible consistency of spatial clustering behaviors across the seismic regions around the world. This work seems to contribute to the idea of a possible worldwide spatial long-range correlation as well as a long-range temporal correlation, i.e., "memory" [22, 25, 4750] between earthquakes temporally apart from each other. In addition, the spatial long-range correlation can be quantified as a scaling exponent through DFA analysis on the time series of earthquake separation distance (spatial interval), whose fluctuation is obviously governed by the seismic region dimension. Such effect may lead to nonuniformity in comparative study across different regions of the world. Here, we develop a size-independent modification of the general DFA method to remove system dimension effect from the fluctuation scaling behavior. This modification is evoked from our analysis of correlations in the Bak-Sneppen model [51], which is one of the simplest SOC models for earthquakes, forest fires, biological evolution, etc.

There is also another theme that appeals to us: is there any universal difference in spatial clustering behavior between shallow and intermediate-depth earthquakes? This doubt is "echoed" with one of the current hot debates in seismology: what constitutes an intermediate-depth earthquake [5255]? It is well-known that the shallow earthquake stems from brittle and ductile behavior of the crust [56]. However, the physical mechanisms behind intermediate-depth earthquakes remain uncertain (two plausible hypothesis: dehydration embrittlement [57] and thermal shear runaway instability $[52,58])$. In particular, the depth cut-off to distinguish between shallow and intermediate-depth earthquakes is still difficult to define [53]. On this account, a depthdependent spatial clustering analysis is implemented with the depth range extending from the shallow seismic region to the intermediate-depth seismic region. One expects that such results may provide valuable clues on detection of the intermediate-depth earthquake mechanisms as well as help explain differences in their seismological behaviors compared to shallow earthquakes.

This paper is organized as follows: In the next section, we review the general DFA method and introduce our modified DFA. In Section 3, the earthquake catalogs and regions are presented. In Section 4, we comparatively analyze the spatial clustering and its depth-dependence with the modified DFA. The last section is devoted to a summary.

\section{Description of the Method}

DFA is a scaling analysis method providing a quantitative representation of the correlation properties of a series of signals. With this method, the long-range correlation can be identified from a nonstationary time series. Such superiority has been confirmed in many research fields [59-63]. The general DFA procedure consists of steps as follows. Let us suppose that $x(i)$ is a length series $N$, and this series is of compact support, i.e., $x(i)=0$, for an nonsignificant fraction of the values only. First, we assume that $x(i)$ are increments of a random walk process around the average $x_{\text {ave }}$ and integrate the time series to acquire the "profile" or "trajectory" of the signal:

$$
y(k)=\sum_{i=1}^{k}\left[x(i)-x_{\text {ave }}\right], \quad i=1, \ldots, N,
$$

where

$$
x_{\text {ave }}=\frac{1}{N} \sum_{k=1}^{N} x(k)
$$

Then $y(k)$ can be viewed as the "displacement" from the origin due to a set of random "walks" $x(i)-x_{\text {ave }}$, because of the integration that the correlations in $x(i)$ get involved. Next, the integrated time series is divided into boxes of equal length $n$. In each box of length, a least-squares line is fit to the data, representing the trend in that box. In DFA, one fits in each box the integrated time series to polynomial function which is the integration of the supposed trend. Here, $y_{n}(k)$ is the fitting polynomial in box $n$. Linear, quadratic, cubic, or higher order polynomials can be used in the fitting procedure (usually called DFA1, DFA2, DFA3, ...). The root-mean-square (rms) fluctuation of this integrated and detrended time series is calculated by

$$
F(n)=\sqrt{\frac{1}{N} \sum_{k=1}^{N}\left[y(k)-y_{n}(k)\right]^{2}} .
$$


Repeating this calculation over all box sizes, we obtain a relationship between $F(n)$ representing the average fluctuation as a function of box size and the box size $n$ representing the number of spikes in a box which is the size of the watch window. If $F(n)$ behaves as a power-law function of $n$, data presents scaling:

$$
F(n) \propto n^{d},
$$

where the scaling exponent $d$ provides information about the type of correlation of the signal. If there is no correlation (white noise), $d=0.5$. Any long-range correlation in the signal will give $d \neq 0.5$. An exponent $d>0.5$ corresponds to positive correlations in the signals, while $d<0.5$ represents negative correlations.

As mentioned above, earthquakes spatial clustering properties can be studied by analyzing the long-range correlations in earthquakes sequences, which are defined in terms of the time series of the separation distance [14]. In this work, we regard the sequence of the earthquakes as a stochastic point process, displaying earthquake which occurs at some random location (hypocenter) in three-dimensional $(3 D)$ space. Such process can be described by Dirac's delta function finite sum to the location $\left(x_{i}^{1}, x_{i}^{2}, x_{i}^{3}\right)$ :

$$
r\left(x^{1}, x^{2}, x^{3}\right)=\sum_{i=1}^{N} \delta\left(x^{1}-x_{i}^{1}, x^{2}-x_{i}^{2}, x^{3}-x_{i}^{3}\right),
$$

$N$ presents the number of earthquake events.

The three-dimensional separation distance between two consecutive earthquakes $i$ and $j$ is measured as Euclidean distance between their hypocenters:

$$
\begin{aligned}
& x_{i}^{1}=\left(R_{0}-h_{i}\right) \cos \theta_{i} \cos \phi_{i}, \\
& x_{i}^{2}=\left(R_{0}-h_{i}\right) \cos \theta_{i} \sin \phi_{i}, \\
& x_{i}^{3}=\left(R_{0}-h_{i}\right) \sin \theta_{i},
\end{aligned}
$$

where the Earth radius is $R_{0}=6.36 \times 10^{6} \mathrm{~m} .\left(\theta_{i}, \phi_{i}\right)$ are the latitude and longitude, in radians. $h_{i}$ is the depth of the hypocenter of $i$ th earthquake in the earthquake data catalog.

$$
\begin{aligned}
& l_{i j}^{a}=x_{i}^{a}-x_{j}^{a}, \quad a=1,2,3, \\
& l_{i j}=\sqrt{\sum_{a=1}^{3}\left(l_{i j}^{a}\right)^{2}} .
\end{aligned}
$$

The range of the location distance in each dimension is then defined as

$$
R \equiv \max \left\{l_{i j}\right\}-\min \left\{l_{i j}\right\} .
$$

As pointed above, earthquakes sequence can be featured by $3 D$ time series of the separation distance $l_{i j}$. The generalization of the DFA to $3 D$ records is straigthforward as one needs only to change the basic equation (3), which is rewritten as

$$
\widetilde{F}(n)=\sqrt{\frac{1}{N} \sum_{k=1}^{N}\left[\mathbf{y}(k)-\mathbf{y}_{n}(k)\right]^{2}}
$$

where $\mathbf{y}(k)=l_{i j}^{1} \boldsymbol{e}_{1}+l_{i j}^{2} \boldsymbol{e}_{2}+l_{i j}^{3} \boldsymbol{e}_{3}$, similarly for the fitting polynomial vector $\mathbf{y}_{n}$. So far one gets the rms fluctuation of the hypocenter separation distance in general DFA method. Considering the range of the location distance, $y(k)$ ("displacement") is the order of $R$. The fitted local trend $\mathbf{y}_{n}(k)$ is expected to have a similar order of magnitude. If the lth order polynomial fitted curve in each box can cancel the $R$ order part in the "displacement", $\widetilde{F}(n)$ will more likely scale with $R^{1 / 2}$. Therefore, the domination of seismic region dimension on the rms fluctuation could be removed by the scaled $\widetilde{F}(n)$. Another considerable scaling component with $R$ is the appearance of earthquake hypocenters, with fractal dimension $D_{f}[12,31,64]$. Thus, more reliable scaling renormalization of $\widetilde{F}(n)$ is

$$
F(n)=\frac{\widetilde{F}(n)}{R^{\left(D_{f}+1 / 2\right)}},
$$

and, for convenience, we chose the single $D_{f}=2.6$ in our calculation [12], although, precisely, $D_{f}$ varies slightly for different seismic areas and depths [64]. Consequently, one can employ this modified DFA to uniformly compare the scaling behaviors of the $l_{i j}$ fluctuation in different earthquake catalogs.

Also, it should be noted that DFA analysis is inappropriate regardless of the order of the polynomial fit applied, because of the presence of nonstationary in time series of the earthquakes separation distance [32]. The detrending of the time series is carried out by the subtraction of the polynomial fits from the "profile"; different order DFA differ in their ability of eliminating trends in the series. For instance, DFA0 can remove just constant trends in the data, DFA1 wipes off constant and linear trends, DFA2 eliminates trends up to second order, and so forth. Thus a comparison of the results obtained from different orders of DFA provides information on the order of trend in the data. In this work, we will present DFA analysis up to the third-order DFA3.

\section{The Regions and Catalogs}

One of the two earthquakes catalogs used in our analysis is derived from the Global Earthquake Catalog, provided by US (United States of America) Geological Survey (USGS), exactly at Advanced National Seismic System [65], in which seismicity from the entire Globe has been recorded. The catalog contains all seismicity completely from January 1, 1972, to December 31, 2016. However, such a limitation in this catalog - that it is not consistent in all regions of the world-merely comprises earthquakes of full magnitudes for the US but only events whose magnitude $\geq 4.5$ (of the Richter scale) for the other areas in the Globe (only if they are informed that the event is clearly felt or causes damage). In seismology, the homogeneity of a global catalog is indispensable with a view of the long-range seismic activity in the world. Thus, in order to obtain homogeneous results from our comparative analysis for different seismic regions over the world, we have screened out only earthquakes with magnitude $\geq 4.5$. The magnitudes $\mathrm{Mb}, \mathrm{ML}, \mathrm{Ms}$, and $\mathrm{Mw}$ are taken into account accordingly and the artificial earthquake 
events (nuclear tests and quarry blasts) have been excluded from the catalog. Finally, 209560 events remain, and $80 \%$ of them happen near the surface of the Earth (depth $\leq 100$ $\mathrm{km}$ ). Besides, to compare our results with previous studies, we adopt the other earthquakes catalog: southern California (SC) earthquakes catalog relocated by Richards-Dinger and Shearer [66], which is widely employed for its high quality relocation.

The regions chosen in our study are all regions of high seismic potential. The Himalayas are among the most seismically frequent regions in the Globe. Most of the earthquakes there are contributed by the Eurasian plates and north-south convergence of the Indian Eurasian plates, the east-west convergence of the Indo-Burmese mountain, and the Indian plate underthrusting below the Eurasian plate [67]. Japan is entangled with huge seismic risk, due to the Pacific-Philippine-Eurasian triple plate junction, where the interactions of three tectonic plates are complex and potential activity is involved [68]. South Asia strides over subduction zone on the eastern side of the India plate, the strike slip structural belt at the back edge of the subduction zone, and the compressed structural zone of the India ocean plate and the pacific plate [69]. The large multicentury earthquakes result from a broad collisional zone where earthquakes with magnitude $\geq 7.0$ are common inland. Most of the shallow major earthquakes and all the other intermediate-depth and deep earthquakes, outside of the Circum-Pacific seismic belt, are distributed here. The Americas (North and South) are surrounded by the Circum-Pacific seismic belt, from the south of Alaska, along the eastern coast of North America, through Canada, California, and western Mexico to Columbia, Peru, and Chile in South America. This wide region is also the most seismically active zone in the world [70]. The geological range of the above regions and corresponding number of earthquake events in our analysis are illustrated in Table 1 and the geographic regional maps are shown in Figure 1.

\section{Results}

Firstly, the spatial clustering properties are analyzed from the separation distance time series of the catalogs. To see whether our modification is valid, we plot $F(n)$ as a function of box size $n$ in Figures 2 and 3, by employing general and modified DFA up to the third order, respectively. The obvious long-range correlation of separation distances can be found with scaling exponents $d$ greater than 0.5 in both Figures 2 and 3. Furthermore, the scaled rms fluctuations in Figure 3 have weak dimensional dependence in contrast with rms fluctuations in Figure 2, which not only verifies our modification satisfactorily but also pretty indicates such long-range correlations come from the earthquake dynamical fluctuations rather than the finite dimension effect. In Figure $3, d$ probably equals $0.70-0.76$, consistent with the previous results of the local seismic regions [41, 71]. The differences of $d$ between the orders of DFA are so small with the interval of uncertainty \pm 0.05 , confirming the precision of our algorithm. Hence, it may be possible for the persistent existence of long-range correlations of separation distances across the Globe as well as in the local seismic regions.
Besides, in Figure 3, scaling exponents $d$ in different catalogs are similar: South Asia: $0.73 \pm 0.02$, Himalayas: $0.70 \pm$ 0.03, America: $0.71 \pm 0.01$, Globe: $0.72 \pm 0.05$, and Japan: $0.76 \pm 0.03$. Such almost identical power-law behaviors of $F(n)$ mean some homogeneity of spatial clustering behavior in worldwide range seems already apparent, at least with the magnitude $\geq 4.5$. Seismologically, this homogeneity of hypocenters spatial clustering could be considered as a manifestation of the fault network structure and the nature of earthquakes long-range interactions across the Globe. Thus, our results are inclined to have a better agreement with the conjecture that the Earth behaves as SOC system with possible spatial long-range relationships between distantly located earthquakes $[5,45,46]$. Meanwhile, what needs to be explained here is that the local spatial clustering [11, 12] cannot be effectively excluded from our $d$ exponent due to the absence of the catalogs declustering procedure as a multitype algorithm widely used in seismology [72]. Specifically, aftershock sequences responsible for local spatial clustering can be identified and removed in declustered catalogs. This means a sufficient work is needed in the future to fully discriminate between long-range or local correlation.

Next, we investigate depth-dependent spatial clustering properties from the catalogs same as above, with the depth ranging from 0 to $230 \mathrm{~km}$. We first divide the depth range into 23 classes of $10 \mathrm{~km}$ each; then we apply the modified DFA analysis on the series of the separation distances $l_{i j}$ belonging to each class. To ensure the efficiency of modified DFA, we check up the adequacy of the 23 depth classes. In Figure 4, we show $d$ as a function of hypocenter depth for different catalogs. From these figures, one can catch sight of the fact that not all the depth classes are filledin for each catalog, due to the inherent event (earthquake) undersampling of the corresponding deep regions in catalogs (Japan and Himalayas). After that, in every catalog, we calculate $d$ for each depth class, associating the value of $d$ with the average depth of the class. As a result, phased nontrivial behaviors of $d$ in Figure 4 can be observed. In the earlier depth range of $0-40 \mathrm{~km}(0-30 \mathrm{~km}$ for Himalayas $), d$ shows a singular behavior irrespective of the catalogs. In this valley-like structure, $d$ plummets from about 0.9 to the assumed minimum value approximately 0.7 in depth: 15.68 $\mathrm{km}$ (South Asia), $15.23 \mathrm{~km}$ (Himalayas), $15.28 \mathrm{~km}$ (America), $15.26 \mathrm{~km}$ (Globe), and $15.59 \mathrm{~km}$ (Japan), respectively. It should be noted that these depths are the average depth of the earthquakes in the class $10-20 \mathrm{~km}$. In order to obtain a possible more precise depth range corresponding to the minimum $d$, the modified DFA analysis as above is repeated with the depth range focused on $0-40 \mathrm{~km}$ and 8 classes of $5 \mathrm{~km}$ each. The higher depth resolution is not considered due to the depth measurement error which results from the fact that recording stations are confined to the Earth's surface; the exact depth of the hypocenter is very hard to accurately resolve. Nonetheless, Figure 5 shows exactly similar valley-like structures of $d$ for different catalogs. The minimum $d$ occurs at depth: $17.85 \mathrm{~km}$ (South Asia), 17.67 $\mathrm{km}$ (Himalayas), $17.76 \mathrm{~km}$ (America), $17.74 \mathrm{~km}$ (Globe), and $17.77 \mathrm{~km}$ (Japan), respectively. Such extremely unanimous depth range $(15-20 \mathrm{~km})$ for minimum $d$ would remind us of 


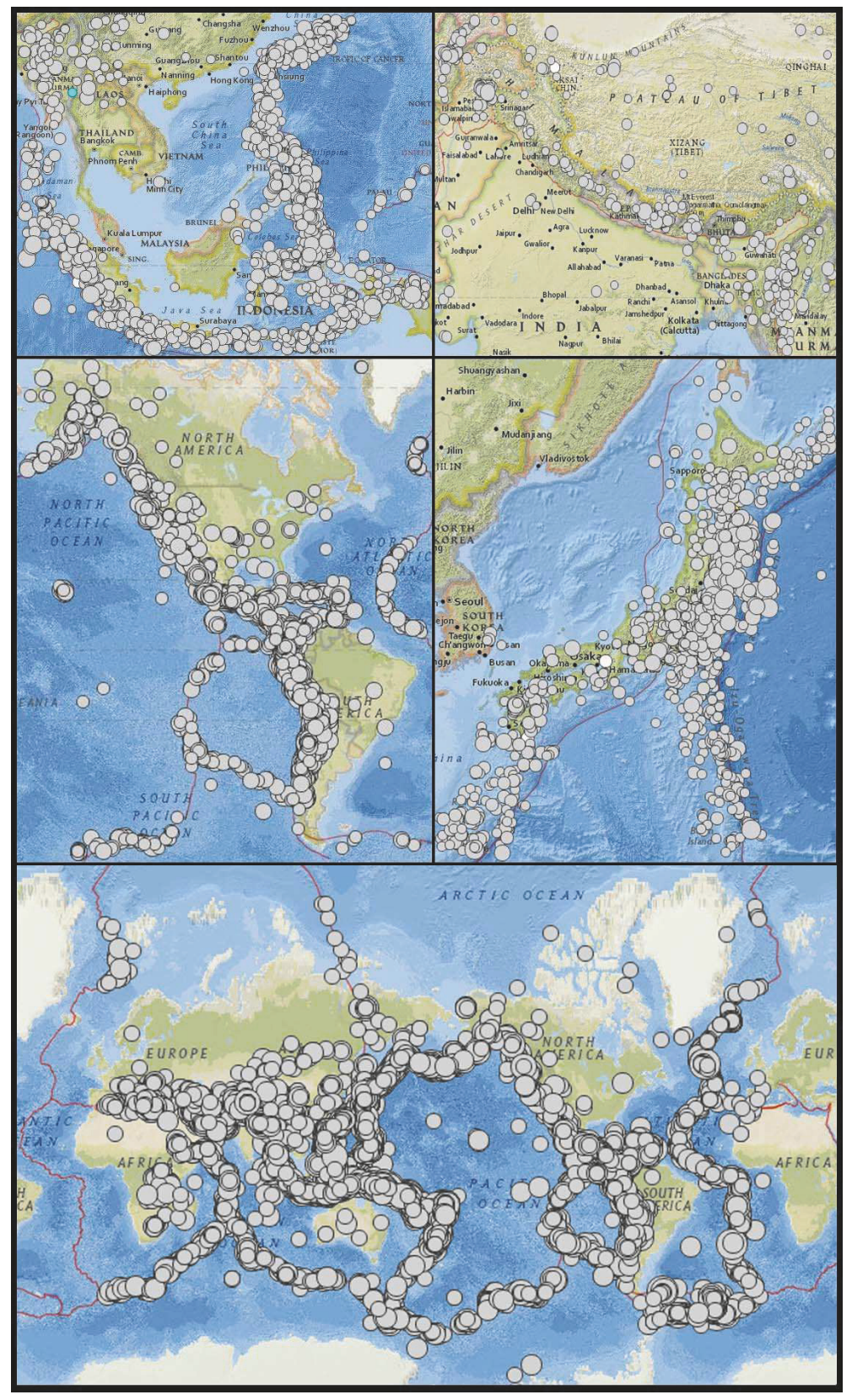

FIgURE 1: Seismic regional maps: South Asia (top-left), Himalayas (top-right), America (middle-left), Japan (middle-right), and Globe (bottom). The gray circles represent the location where earthquake occurs frequently.

TABLE 1: Geological range (latitude and longitude) and earthquake event number of the regions.

\begin{tabular}{|c|c|c|c|c|c|}
\hline Seismic Region & Min Lat & Max Lat & Min Lng & Max Lng & Earthquake number \\
\hline Globe & -90 & 90 & -180 & 180 & 209560 \\
\hline Japan & 25.73 & 47.83 & 126.43 & 148.15 & 18275 \\
\hline America & -57.32 & 71.59 & -168.05 & -33.04 & 36628 \\
\hline Himalayas & 20.37 & 38.14 & 70.35 & 98.03 & 5895 \\
\hline South Asia & -10.06 & 28.26 & 92.16 & 140.08 & 70966 \\
\hline
\end{tabular}




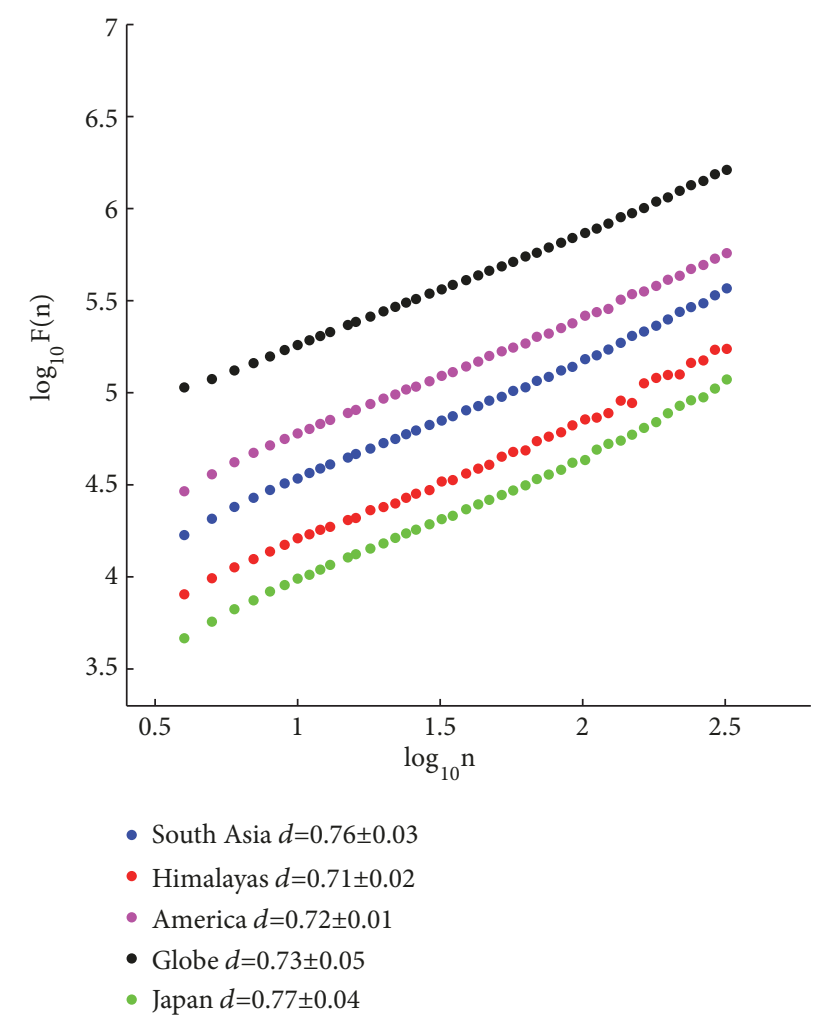

Figure 2: $\log _{10} F(n)-\log _{10} n$ plots for the time series of hypocenter separation distance in catalogs: South Asia, Himalayas, America, Globe, and Japan. The employed method for calculations is the general DFA.

whether a determined universal depth range corresponding to the minimum $d$ exists. To further prove this, we apply the same calculation to the SC catalog, relocated by RichardsDinger (2000). The small depth error $(741 \mathrm{~m})$ in this catalog enables us to analyze $d$ in $1 \mathrm{~km}$ class. In Figure 6, the valleylike structure exhibits again, associating a minimum $d$ at the depth $15.49 \mathrm{~km}$. More interestingly, in Telesca's results from SC catalog [36], the scaling exponent of temporal clustering shows a minimum corresponding to the depth range of 15-16 $\mathrm{km}$. Worthy of note is that similar range of $n$ has been used to calculate $d$ in Figures 4-6 and each $F(n)$ as a function of $n$ presents a good scaling. Although an exhaustive study should be further performed, it seems that the spatial clustering, as well as the temporal clustering of shallow earthquakes, shows a minimum $d$ corresponding to the depth of $15-20$ $\mathrm{km}$. This minimum could be derived from the brittle and ductile behavior of the crust versus depth, as presented in the synoptic shear zone model [73]. Therefore, we argue that the clustering behavior as a typical seismic dynamic evolution may be characterized with spatiotemporal consistency.

One can further find in Figure 4 that $d$ sustains falling with the increasing depth in all catlogs, until the next nontrivial depth region is approaching, and in each catalog $d$ almost gradually flattens after depth $50 \mathrm{~km}$. It is worth noting that 50 $\mathrm{km}$ is the depth at which well-developed aftershock sequences become infrequent [74] and also the maximum depth of

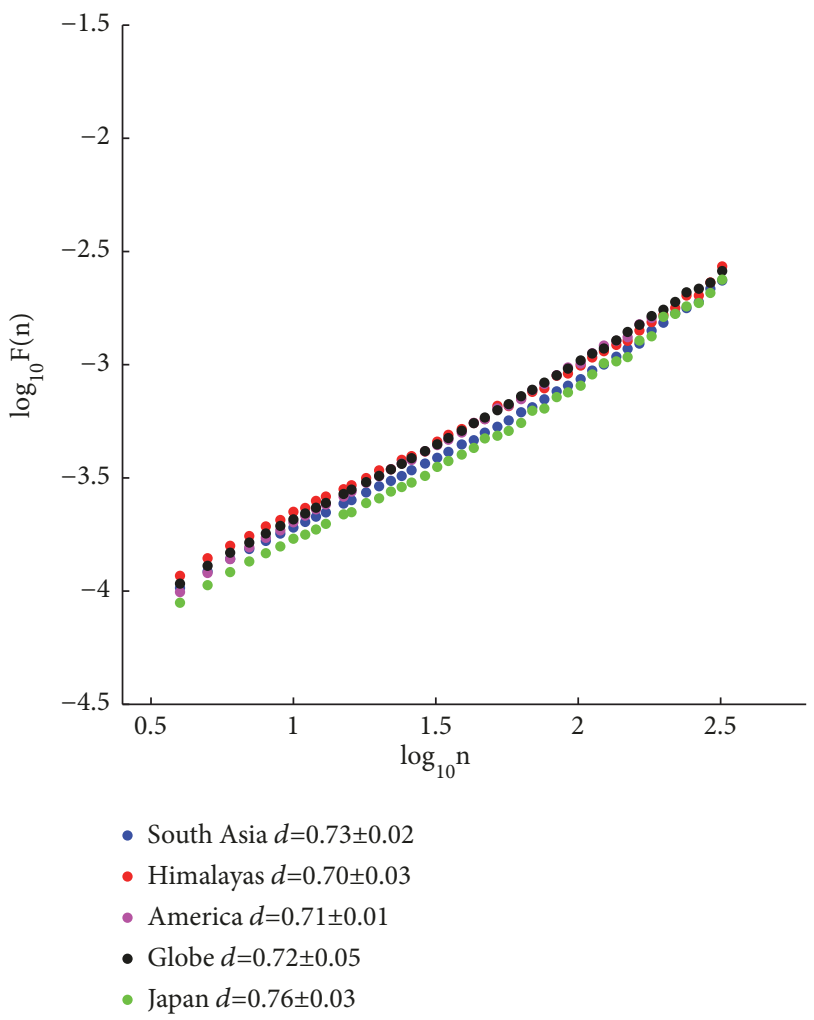

FIGURE 3: $\log _{10} F(n)-\log _{10} n$ plots for the time series of hypocenter separation distance in catalogs: South Asia, Himalayas, America, Globe, and Japan. The employed method for calculations is the modified DFA.

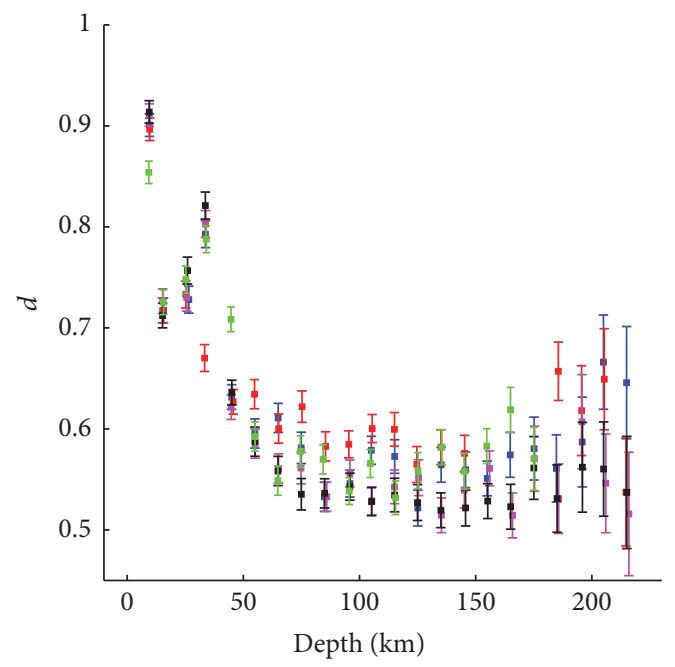

$$
\begin{aligned}
& \text { - South Asia } \\
& \text { - Himalayas } \\
& \text { - America } \\
& \text { - Globe } \\
& \text { - Japan }
\end{aligned}
$$

FIGURE 4: Variation with the depth of $d$, calculated by the modified DFA for the time series of hypocenter separation distance in catalogs: South Asia, Himalayas, America, Globe, and Japan. Depth range is $0-230 \mathrm{~km}$ in $10 \mathrm{~km}$ class. 

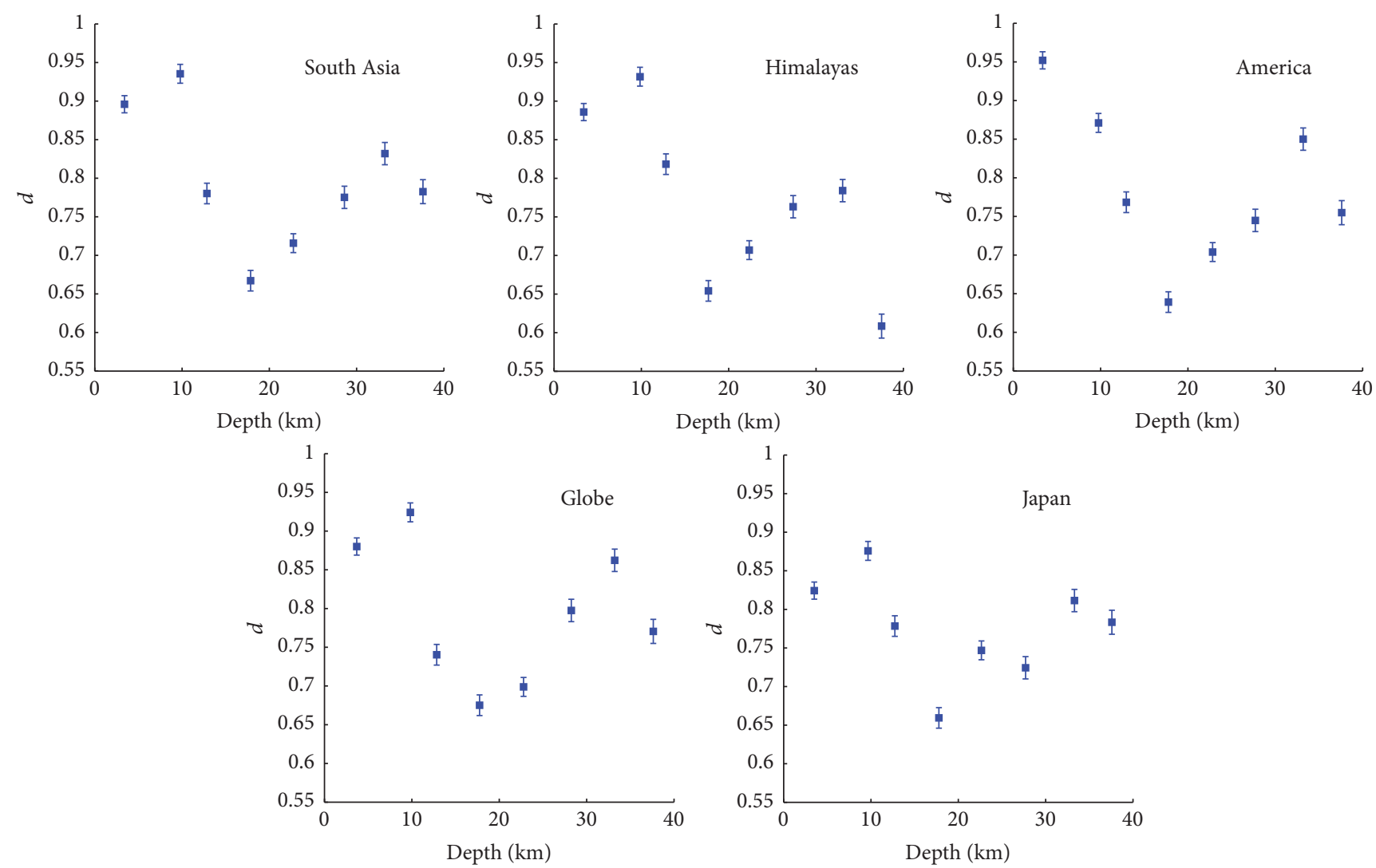

Figure 5: Variation with the depth of $d$, calculated by the modified DFA for the time series of hypocenter separation distance in catalogs: South Asia, Himalayas, America, Globe, and Japan. Depth range is $0-40 \mathrm{~km}$ in $5 \mathrm{~km}$ class.

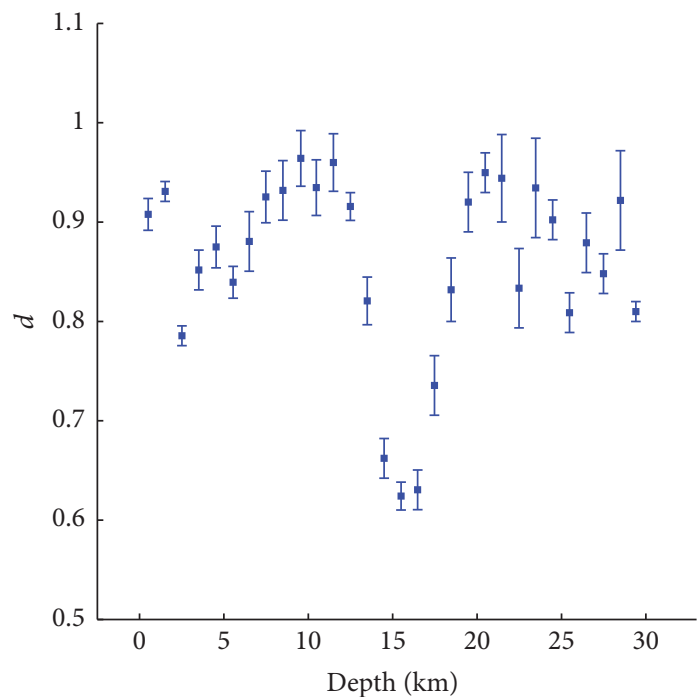

FIGURE 6: Variation with the depth of $d$, calculated by the modified DFA for the time series of hypocenter separation distance in SC catalog relocated by Richards-Dinger and Shearer.

interplate subduction zone earthquakes [75]. Either of the above analogies accounts for the weakening long-term correlations in deep earthquakes. After transition depth $(50 \mathrm{~km})$, exactly beyond the depths $54.98 \mathrm{~km}$ (South Asia), $55.09 \mathrm{~km}$ (America), $64.88 \mathrm{~km}$ (Japan), $65.19 \mathrm{~km}$ (Himalayas), and $74.94 \mathrm{~km}$ (Globe), $d$ presents smooth fluctuations ranging within $0.52-0.62$. Such "smoothness" indicates the presence of tendency to flicker-noise type dynamics in the earthquake process $[36,71]$. On the other hand, these closely related but not exactly the same depths may correspond to the fact that it is hard to define a depth cut-off to distinguish between shallow and intermediate-depth earthquakes. For example, Wadati (1929) defines shallow earthquake as $\geq 60 \mathrm{~km}$ and intermediate-depth earthquake as 100 - 200 [76]; Gutenberg and Richter (1949) define intermediate-depth earthquake as 70 - 300 [77]; Frohlich (2006) and Houston (2007) define intermediate-depth earthquake as $50-300 \mathrm{~km}[74,78]$. These inconsistent definitions as well as our results imply that shallow and intermediate-depth earthquake dynamic mechanisms may not be differentiable at least in the depth range $50-70 \mathrm{~km}$. This puzzle, as one can see, remains, to us, regrettable but predictable for two conceivable reasons: one is the authentic existence of strong interactions between the occurrence of intermediate-depth and shallow earthquakes in recent seismological research [79]; the other is the disparate seismotectonics between seismic regions, e.g., Himalayas dominated by the shallow earthquakes but the South Asia frequently invaded by intermediate-depth and deep earthquakes. Nevertheless, the significant and 
synchronized suppression of $d$ in different catalogs shows that the spatial clustering of intermediate-depth earthquakes is widely different from that of shallow earthquakes. These low and stable spatial clustering properties in intermediate-depth earthquakes may originate from two plausible hypotheses: dehydration embrittlement and thermal shear runaway instability. Based on them, a particular kind of intermediate-depth earthquakes clustering could be regarded as a earthquake nest [80] which is isolated from nearby seismic activity [53]. Such nest mode would lead to very small rupture regions and/or slow rupture velocities compared with shallow earthquakes $[54,55]$. In a word, the spatial long-range correlations (perhaps the temporal ones as well) may become strong weakening in intermediate-depth earthquakes, relative to a cascading thermal shear runaway, with a leading inefficient radiation process followed by a quick and active efficient rupture [81, 82]. Meanwhile, in Figure 4, the error and instability of $d$ are distinct beyond the depth about $170 \mathrm{~km}$, associating with the event undersampling in catalogs. Even so, any uniform earthquakes long-range dynamic mechanisms in such deeper regions could not be precluded on account of our results, because some possible earthquake nests may be found or confirmed as the extensive regional and global networks [53]. Although our results are not sufficient, it may open up a intriguing prospect for the exploration of the dynamic correlation mechanisms in seismicity.

\section{Summary}

Analyzing the earthquakes clustering behaviors gains insight into the underlying dynamics of seismicity. In the present paper, we introduced a modified version of general DFA method to demonstrate the extremely similar spatial clustering behaviors for different wide seismic regions over the world. Some kind of homogeneity involved worldwide in the spatial dynamic seismic evolution is shown.

Moreover, the depth-dependent spatial clustering properties have been identified on the comparison of different catalogs. A phased nontrivial clustering behavior has been revealed from shallow to intermediate-depth earthquakes. In the shallow regions, $d$ has been identified with a minimum corresponding to the depth range of $15-20$ $\mathrm{km}$. Comparing with the previous results in the temporal clustering analysis, we argue that a consistent characteristic depth range may exist in both spatial and temporal clustering behaviors of shallow earthquakes. In the intermediatedepth regions, earthquakes spatial clustering has been found faded with the much lower and stable value of $d$, which means the strong weakening spatial long-range correlations in intermediate-depth earthquakes compared to shallow earthquakes, probably due to the earthquake nest mechanisms.

We should admit that our results could not preclude other clustering behaviors in intermediate-depth earthquakes, because the extensive regional and global networks of earthquake nests may be truly natural. Such evidence may be supplied by more comprehensive statistical correlation analysis of seismic time series. We hope to report our progress in this regard in the future.

\section{Data Availability}

The data used to support the findings of this study are available from the corresponding author upon request.

\section{Conflicts of Interest}

The authors declare that they have no conflicts of interest.

\section{Acknowledgments}

This work is supported by the NSFC under Grants Nos. 11305143 and 41230637.

\section{References}

[1] http://www.nature.com/nature/debates/earthquake.

[2] G. A. Prieto, "Imaging the Deep Earth," Science, vol. 338, no. 6110, pp. 1037-1038, 2012.

[3] B. Gutenberg and C. F. Richter, "Earthquake magnitude, intensity, energy, and acceleration," Bulletin of the Seismological Society of America, vol. 32, no. 3, pp. 163-191, 1942.

[4] F. Omori and Coll., "On the Aftershocks of Earthquakes," Journal of the College of Science, Imperial University of Tokyo, vol. 7, pp. 111-120, 1894.

[5] P. Bak, C. Tang, and K. Wiesenfeld, "Self-organized criticality: an explanation of the $1 /$ f noise," Physical Review Letters, vol. 59, no. 4, pp. 381-384, 1987.

[6] H. Kawamura, T. Hatano, N. Kato, S. Biswas, and B. K. Chakrabarti, "Statistical physics of fracture, friction, and earthquakes," Reviews of Modern Physics, vol. 84, no. 2, pp. 839-884, 2012.

[7] D. Sornette and M. J. Werner, "Seismicity, statistical physics approaches to," in Extreme Environmental Events, R. Meyers, Ed., Springer, New York, NY, USA, 2011.

[8] L. de Arcangelis, C. Godano, J. R. Grasso, and E. Lippiello, "Statistical physics approach to earthquake occurrence and forecasting," Physics Reports, vol. 628, pp. 1-91, 2016.

[9] Y. Kamer, G. Ouillon, D. Sornette, and J. Wössner, "Condensation of earthquake location distributions: Optimal spatial information encoding and application to multifractal analysis of south Californian seismicity," Physical Review E: Statistical, Nonlinear, and Soft Matter Physics, vol. 92, no. 2, Article ID 022808, 2015.

[10] L. de Arcangelis, C. Godano, E. Lippiello, and M. Nicodemi, "Universality in Solar Flare and Earthquake Occurrence," Physical Review Letters, vol. 96, no. 5, Article ID 051102, 2006.

[11] E. Lippiello, L. de Arcangelis, and C. Godano, "Role of Static Stress Diffusion in the Spatiotemporal Organization of Aftershocks," Physical Review Letters, vol. 103, no. 3, 2009.

[12] M. Bottiglieri, E. Lippiello, C. Godano, and L. de Arcangelis, "Identification and spatiotemporal organization of aftershocks," Journal of Geophysical Research: Solid Earth, vol. 114, no. B3, Article ID B03303, 2009.

[13] L. Telesca, G. Babayev, and F. Kadirov, “Temporal clustering of the seismicity of the Absheron-Prebalkhan region in the Caspian Sea area," Natural Hazards and Earth System Sciences, vol. 12, no. 11, pp. 3279-3285, 2012.

[14] A. Corral, "Long-term clustering, scaling, and universality in the temporal occurrence of earthquakes," Physical Review Letters, vol. 92, Article ID 108501, 2004. 
[15] J. R. Holliday, J. B. Rundle, D. L. Turcotte, W. Klein, K. F. Tiampo, and A. Donnellan, "Space-time clustering and correlations of major earthquakes," Physical Review Letters, vol. 97, no. 23, Article ID 238501, 2006.

[16] I. Zaliapin, A. Gabrielov, V. Keilis-Borok, and H. Wong, "Clustering Analysis of Seismicity and Aftershock Identification," Physical Review Letters, vol. 101, no. 1, 2008.

[17] J. Davidsen, G. Kwiatek, and G. Dresen, "No evidence of magnitude clustering in an aftershock sequence of nano- and picoseismicity," Physical Review Letters, vol. 108, no. 3, Article ID 038501, 2012.

[18] Y. Ashkenazy, P. C. Ivanov, S. Havlin, C. Peng, A. L. Goldberger, and H. E. Stanley, "Magnitude and Sign Correlations in Heartbeat Fluctuations," Physical Review Letters, vol. 86, no. 9, pp. 1900-1903, 2001.

[19] S. Lennartz, V. N. Livina, A. Bunde, and S. Havlin, "Long-term memory in earthquakes and the distribution of interoccurrence times," EPL (Europhysics Letters), vol. 81, no. 6, Article ID 69001, p. $69001,2008$.

[20] H. E. Stanley, L. A. N. Amaral, A. L. Goldberger, S. Havlin, P. C. Ivanov, and C. K. Peng, "Statistical physics and physiology: monofractaland multifractal approaches," Physica A: Statistical Mechanics and its Applications, vol. 270, pp. 309-324, 1999.

[21] D. W. Steeples and D. Steeples, "Far-Field aftershocks of the 1906 earthquake," Bulletin of the Seismological Society of America, vol. 86, no. 4, pp. 921-924, 1996.

[22] J. N. Tenenbaum, S. Havlin, and H. E. Stanley, "Earthquake networks based on similar activity patterns," Physical Review E: Statistical, Nonlinear, and Soft Matter Physics, vol. 86, no. 4, Article ID 046107, 2012.

[23] L. Telesca, V. Lapenna, and M. Macchiato, "Multifractal fluctuations in earthquake-related geoelectrical signals," New Journal of Physics, vol. 7, article 214, 2005.

[24] F. C. Lin, M. H. Ritzwoller, Y. Yang, M. P. Moschetti, and M. J. Fouch, "Complex and variable crustal and uppermost mantle seismic anisotropy in the western United States," Nature Geoscience, vol. 4, pp. 55-61, 2011.

[25] Z. Zheng, K. Yamasaki, J. Tenenbaum, B. Podobnik, Y. Tamura, and H. E. Stanley, "Scaling of seismic memory with earthquake size," Physical Review E: Statistical, Nonlinear, and Soft Matter Physics, vol. 86, no. 1, Article ID 011107, 2012.

[26] T. R. K. Mohan and P. G. Revathi, "Earthquake correlations and networks: a comparative study," Physical Review E: Covering Statistical, Nonlinear, Biological, and Soft Matter Physics, vol. 83, no. 4, Article ID 046109, 2011.

[27] J. Davidsen and A. Green, "Are earthquake magnitudes clustered?" Physical Review Letters, vol. 106, no. 10, Article ID 108502, 2011.

[28] T. P. Peixoto, D. Katharina, and D. Jorn, "Spatiotemporal correlations of aftershock sequences," Journal of Geophysical Research: Solid Earth, vol. 115, no. B10, 2010.

[29] E. Lippiello, L. de Arcangelis, and C. Godano, "Influence of time and space correlations on earthquake magnitude," Physical Review Letters, vol. 100, no. 3, Article ID 038501, 2008.

[30] B. Nana and P. Woafo, "Synchronization in a ring of four mutually coupled van der Pol oscillators: theory and experiment," Physical Review E: Statistical, Nonlinear, and Soft Matter Physics, vol. 74, no. 4, Article ID 046213, 2006.

[31] M. Baiesi and M. Paczuski, "Scale-free networks of earthquakes and aftershocks," Physical Review E: Statistical, Nonlinear, and Soft Matter Physics, vol. 69, no. 6, Article ID 066106, 2004.
[32] Z. Chen, P. C. H. Ivanov, K. Hu, and H. E. Stanley, "Effect of nonstationarities on detrended uctuation analysis," Physical Review E: Statistical, Nonlinear, and Soft Matter Physics, vol. 65, no. 4, part 1, Article ID 041107, 2002.

[33] Y. Y. Kagan, “Are earthquakes predictable?" Geophysical Journal International, vol. 131, no. 3, pp. 505-525, 1997.

[34] C.-K. Peng, S. Havlin, H. E. Stanley, and A. L. Goldberger, "Quantification of scaling exponents and crossover phenomena in nonstationary heartbeat time series," Chaos: An Interdisciplinary Journal of Nonlinear Science, vol. 5, no. 1, pp. 82-87, 1995.

[35] L. Telesca, V. Cuomo, V. Lapenna, and M. Macchiato, "Intermittent-type temporal fluctuations in seismicity of the Irpinia (Southern Italy) region," Geophysical Research Letters, vol. 28, no. 19, pp. 3765-3768, 2001.

[36] L. Telesca, V. Cuomo, and V. Lapenna, "Depth-dependent time-clustering behaviour in seismicity of Southern California," Geophysical Research Letters, vol. 28, no. 22, pp. 4323-4326, 2001.

[37] L. Telesca and V. Lapenna, "Long-range correlations in twodimensional spatio-temporal seismic fluctuations," Physica A: Statistical Mechanics and its Applications, vol. 377, no. 1, pp. 279284, 2007.

[38] L. Telesca, V. Lapenna, and M. Macchiato, "Multifractal fluctuations in earthquake-related geoelectrical signals," New Journal of Physics, vol. 7, article 214, 2005.

[39] P. A. Varotsos, N. V. Sarlis, and E. S. Skordas, "Temporal correlations between the earthquake magnitudes before major mainshocks in Japan," 2017, https://arxiv.org/abs/1612.07032.

[40] L. Telesca, G. Colangelo, V. Lapenna, and M. Macchiato, "Fluctuation dynamics in geoelectrical data: an investigation by using multifractal detrended fluctuation analysis," Physica A: Statistical Mechanics and its Applications, vol. 332, no. 5-6, pp. 398-404, 2004.

[41] L. A. Martin-Montoya, N. M. Aranda-Camachob, and C. J. Quimbay, "Long-range correlations and trends in Colombian seismic time series," Physica A: Statistical Mechanics and its Applications, vol. 421, pp. 124-133, 2015.

[42] E. L. Flores-Marquez, A. Ramirez-Rojas, and L. Telesca, "Multifractal detrended fluctuation analysis of earthquake magnitude series of Mexican South Pacific Region," Applied Mathematics and Computation, vol. 265, pp. 1106-1114, 2015.

[43] L. Telesca and L. Toth, "Multifractal detrended fluctuation analysis of Pannonian earthquake magnitude series," Physica A: Statistical Mechanics and its Applications, vol. 448, pp. 21-29, 2016.

[44] X. X Fan and M. Lin, "Multiscale multifractal detrended fluctuation analysis of earthquake magnitude series of Southern California," Physica A: Statistical Mechanics and its Applications, vol. 479, pp. 225-235, 2017.

[45] D. Ferreira, A. Papa, and R. Menezes, "On the agreement between small-world-like OFC model and real earthquakes," Physica A: Statistical Mechanics and its Applications, vol. 379, no. 7, pp. 669-675, 2015.

[46] D. Ferreira, J. Ribeiro, A. Papa, and R. Menezes, "Towards evidence of long-range correlations in shallow seismic activities," EPL (Europhysics Letters), vol. 121, no. 5, Article ID 58003, 2018.

[47] E. Lippiello, L. de Arcangelis, and C. Godano, "Memory in selforganized criticality," EPL (Europhysics Letters), vol. 72, no. 4, article 678, 2005.

[48] V. N. Livina, S. Havlin, and A. Bunde, "Memory in the occurrence of earthquakes," Physical Review Letters, vol. 95, no. 20, Article ID 208501, 2005. 
[49] H. Kawamura, Y. Ueda, S. Kakui, S. Morimoto, and T. Yamamoto, "Statistical properties of the one-dimensional Burridge-Knopoff model of earthquakes obeying the rate- and state-dependent friction law," Physical Review E: Statistical, Nonlinear, and Soft Matter Physics, vol. 95, no. 4, Article ID 042122, 14 pages, 2017.

[50] N. V. Sarlis, E. S. Skordas, and P. A. Varotsos, "Nonextensivity and natural time: the case of seismicity," Physical Review E: Statistical, Nonlinear, and Soft Matter Physics, vol. 82, no. 2, Article ID 021110, 2010.

[51] K. Ma, C. B. Yang, and X. Cai, "Correlations in the BakSneppen model from detrended fluctuation analysis," Physica A: Statistical Mechanics and its Applications, vol. 357, no. 3-4, pp. 455-465, 2005.

[52] P. B. Keleman and G. Hirth, "A periodic shear-heating mechanism for intermediate-depth earthquakes in the mantle," Nature, vol. 446, no. 7137, pp. 787-790, 2007.

[53] G. A. Prieto, G. C. Beroza, S. A. Barrett, G. A Lopez, and M. Florez, "Earthquake nests as natural laboratories for the study of intermediate-depth earthquake mechanics," Tectonophysics, vol. 570-571, pp. 42-56, 2012.

[54] P. Poli and G. A. Prieto, "Global rupture parameters for deep and intermediate-depth earthquakes," Journal of Geophysical Research: Solid Earth, vol. 121, pp. 8871-8887, 2016.

[55] P. Poli, G. A. Prieto, E. Rivera, and S. Ruiz, "Earthquakes initiation and thermal shear instability in the Hindu Kush intermediate depth nest," Geophysical Research Letters, vol. 43, no. 4, pp. 1537-1542, 2016.

[56] J. C. Jaeger and N. G. W. Cook, Fundamentals of Rock Mechanics, Chapman and Hall, London, UK, 1979.

[57] H. W. Green and H. Houston, "The Mechanics of Deep Earthquakes," Annual Review of Earth and Planetary Sciences, vol. 23, pp. 169-213, 1995.

[58] G. A. Prieto, M. Florez, S. A. Barrett et al., "Seismic evidence for thermal runaway during intermediate-depth earthquake rupture," Journal of Geophysical Research Letters, vol. 40, no. 23, pp. 6064-6068, 2013.

[59] F. von Wegner, H. Laufs, and E. Tagliazucchi, "Mutual information identifies spurious Hurst phenomena in resting state EEG and fMRI data," Physical Review E: Statistical, Nonlinear, and Soft Matter Physics, vol. 97, no. 2, Article ID 022415, 2018.

[60] Y. H. Wu, W. L. Wang, N. Yan, and B. Wei, "Experimental investigations and phase-field simulations of triple-phase-separation kinetics within liquid ternary $\mathrm{Co}-\mathrm{Cu}-\mathrm{Pb}$ immiscible alloys," Physical Review E: Covering Statistical, Nonlinear, Biological, and Soft Matter Physics, vol. 95, no. 5, Article ID 052111, 2017.

[61] R. J. Buonocore, T. Aste, and T. D. Matteo, "Asymptotic scaling properties and estimation of the generalized Hurst exponents in financial data," Physical Review E: Covering Statistical, Nonlinear, Biological, and Soft Matter Physics, vol. 95, no. 4, Article ID 042311, 2017.

[62] D. Das, K. Shiladitya, K. Biswas et al., "Wavelet-based multiscale analysis of bioimpedance data measured by electric cellsubstrate impedance sensing for classification of cancerous and normal cells," Physical Review E: Statistical, Nonlinear, and Soft Matter Physics, vol. 92, no. 6, Article ID 062702, 2015.

[63] Z. C. Zhang, Y. J. Zhang, D. H. Shen, and W. Zhang, "The dynamic cross-correlations between mass media news, new media news, and stock returns," Complexity, vol. 2018, Article ID 7619494, p. 11, 2018.

[64] Y. Y. Kagan, "Fractal dimension of brittle fracture," Journal of Nonlinear Science, vol. 1, no. 1, pp. 1-16, 1991.
[65] http://quake.geo.berkeley.edu/.

[66] K. B. Richards-Dinger and P. M. Shearer, "Earthquake locations in southern California obtained using source-specific station terms," Journal of Geophysical Research, vol. 105, no. B5, pp. 10,939-10,960, 2000.

[67] S. Srilakshmi and R. K. Tiwari, "Are northeast and western Himalayas earthquake dynamics better 'organized' than Central Himalayas: an artificial neural network approach," Geofísica Internacional, vol. 46, no. 1, pp. 63-73, 2007.

[68] M. Mahdyiar, "Modeling uncertainty in earthquake occurrence in Japan," AIR Worldwide Corporation (AIR), Tech. Rep., http://www.air-worldwide.com/Publications/AIR-Currents/ attachments/AIR-Currents-Japan-Quake/, February 2009.

[69] C. Vigny, W. Simons, S. Abu, and B. A. c. Ambrosius, "Insight into the 2004 Sumatra-Andaman earthquake from GPS measurements in southeast Asia," Nature, vol. 436, no. 7048, 2005.

[70] R. Trenkamp, J. N. Kellogg, J. T. Freymueller, and H. P. Mora, "Wide plate margin deformation, southern Central America and northwestern South America, CASA GPS observations," Journal of South American Earth Sciences, vol. 15, no. 2, pp. 157171, 2002.

[71] M. Fukamachi and T. Nagatani, "Sidle effect on pedestrian counter flow," Physica A: Statistical Mechanics and its Applications, vol. 377, no. 1, pp. 269-278, 2007.

[72] T. V. Stiphout, J. Zhuang, and D. Marsan, "Seismicity declustering," in Community Online Resource for Statistical Seismicity Analysis, 2012.

[73] C. H. Scholz, The Mechanics of Earthquakes And Faulting, Cambridge University Press, Cambridge, UK, 1990.

[74] C. Frohlich, Deep earthquakes, Cambridge University Press, Cambridge, UK, 2006.

[75] S. L. Bilek, T. Lay, and L. J. Ruff, "Radiated seismic energy andearthquake source duration variations from teleseismic source time functionsfor shallow subduction zone thrust earthquake," Journal of Geophysical Research: Solid Earth, vol. 109, no. B9, Article ID B09308, 2004.

[76] K. Wadati, "Shallow and deep earthquakes," Geophysical Magzine, vol. 2, pp. 1-36, 1929.

[77] B. Gutenberg and C. Richter, in Seismicity of The Earth and Associated Phenomena, vol. 310, Princeton University Press, Princeton, NJ, USA, 1949.

[78] H. Houston, “Deep Earthquakes," Treatise on Geophysics, vol. 4, pp. 321-350, 2007.

[79] J. Jara, A. Socquet, D. Marsan, and M. Bouchon, "Long-term interactions between intermediate depth and shallow seismicity in North Chile subduction zone," Geophysical Research Letters, vol. 44, no. 18, pp. 9283-9292, 2017.

[80] Z. Zarifi and J. Havskov, "Characteristics of dense nests of deep and intermediate-depth seismicity," Advances in Geophysics, vol. 46, no. C, pp. 237-278, 2003.

[81] P. Poli, G. A. Prieto, C. Q. Yu, M. Florez, and H. Agurtodetzel, "Complex rupture of the M6.3 2015 March 10 Bucaramanga earthquake: evidence of strong weakening process," Geophysical Journal International, vol. 205, pp. 988-994, 2016.

[82] G. A. Prieto, M. Florez, S. A Barrett, G. C. Beroza, and P. Pedraza, "Seismic evidence for thermal runaway during intermediate-depth earthquake rupture," Geophysical Research Letters, vol. 40, no. 23, pp. 6064-6068, 2013. 


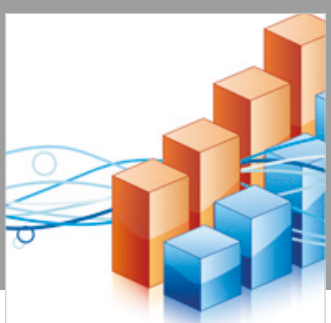

Advances in

Operations Research

\section{-n-m}
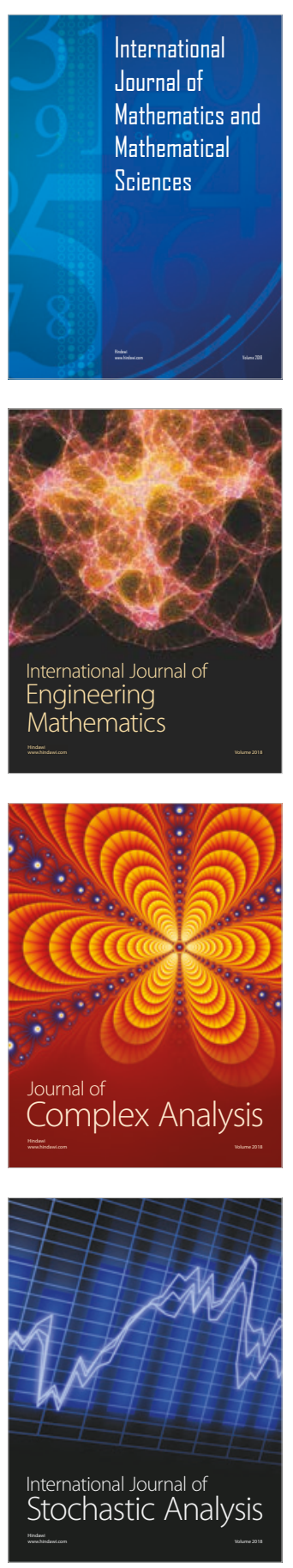
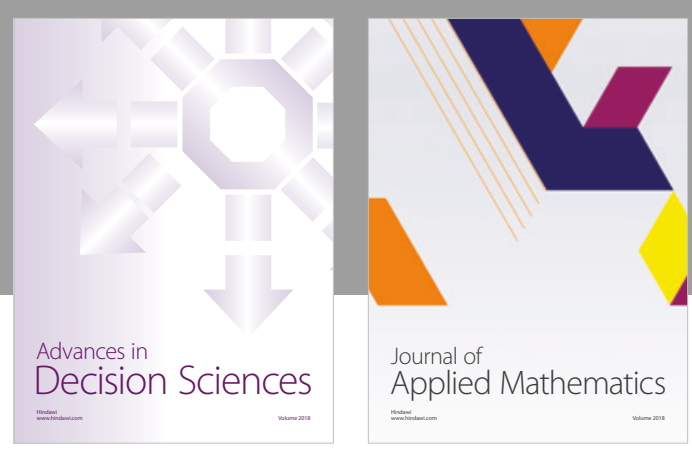

Journal of

Applied Mathematics
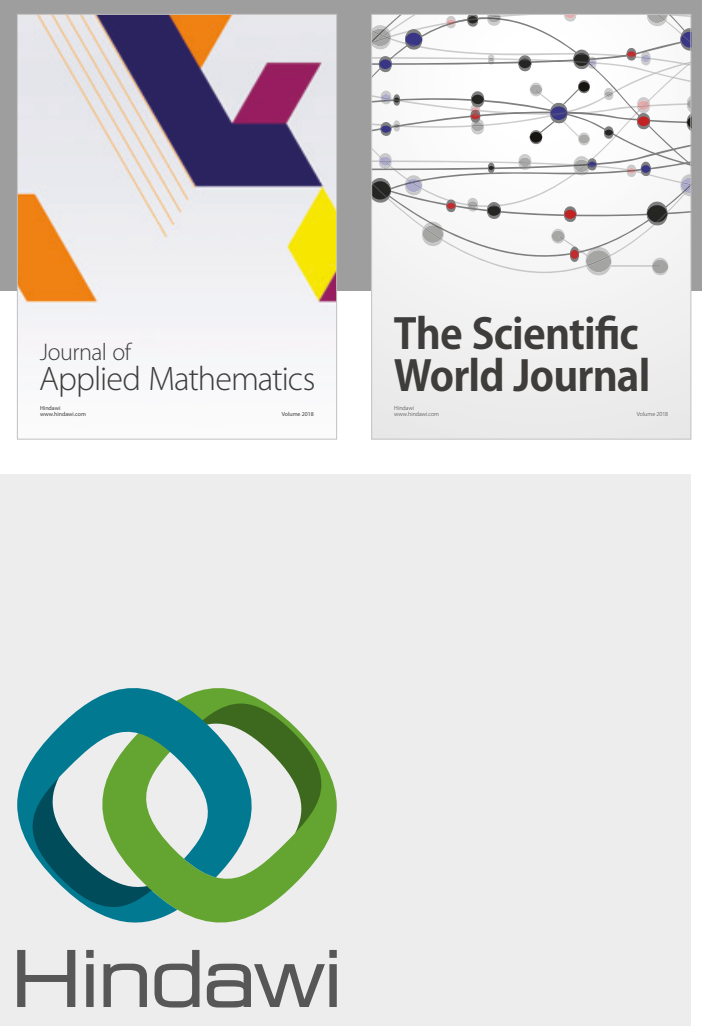

Submit your manuscripts at

www.hindawi.com

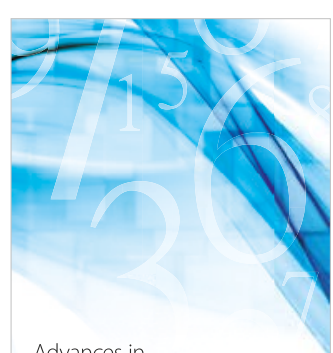

Advances in
Numerical Analysis
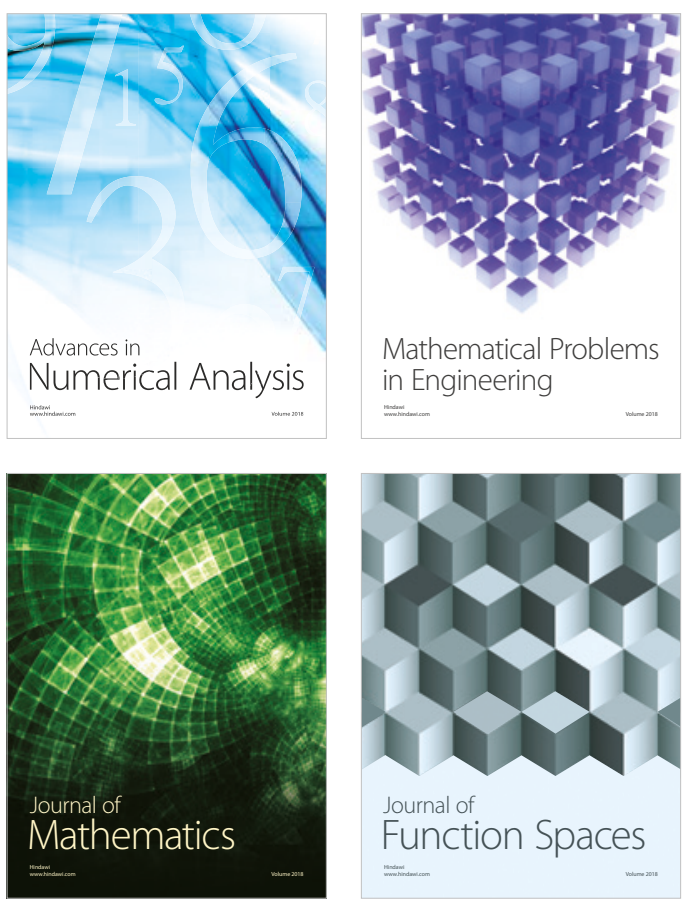

Mathematical Problems in Engineering

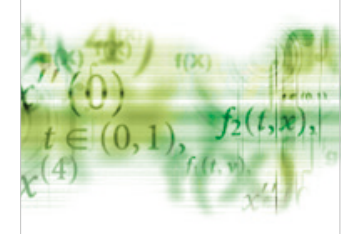

International Journal of

Differential Equations

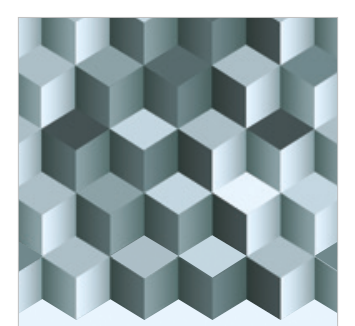

Journal of

Function Spaces
The Scientific

World Journal

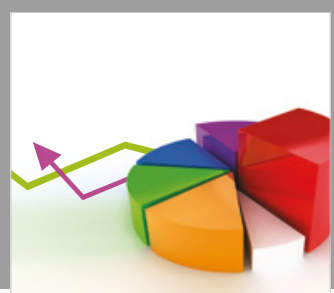

Journal of

Probability and Statistics
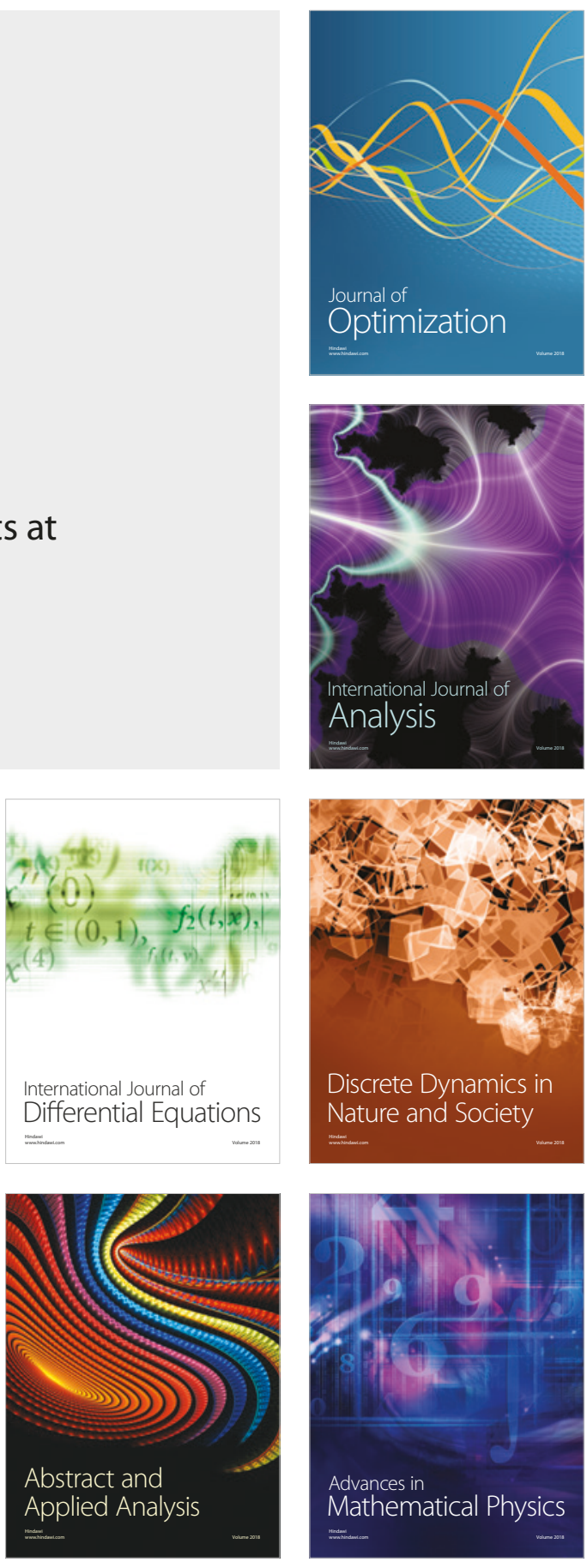